\title{
CAPÍTULO VIII
}

\section{LA PYME Y EL DISTINTIVO DE EMPRESA SOCIALMENTE RESPONSABLE (ESR): RAZONES PARA PERDER LA CERTIFICACIÓN DE CEMEFI}

\author{
Elia Carmina Cota Montes \\ Maestra en Administración, docente de la Universidad Autónoma de Occidente, México. \\ Doctorante del Doctorado en Innovación y Administración de las Organizaciones y la \\ Responsabilidad Social Empresaria (RSE) en la PyME. Líneas de investigación: Correo \\ electrónico: elia_cota@hotmail.com.
}

\section{Ruth Norma Hamasaki Gálvez}

Doctora en Estudios Organizacionales. Investigadora y consultora de PyMES y la RSE de la agencia de consultoría Strategio, S.C. Miembro del Consejo de Investigación Social y Empresarial de Sinaloa (CADISE), México. Correo electrónico: ruthamasaki@hotmail.com.

\section{Resumen}

El estudio sobre las pequeñas empresas ha sido desde hace tiempo un tema relevante desde diversas disciplinas, por la importancia que este tipo de organizaciones tiene en el ámbito social y el desarrollo económico de los países. Sin embargo, aún se requiere ampliar su conocimiento respecto a temas como la competitividad, la innovación, la calidad y en particular sobre la Responsabilidad Social Empresarial (RSE); considerando que ésta última asume implicaciones más amplias sobre los impactos de las operaciones empresariales en su medio ambiente. El surgimiento de la sustentabilidad y el cuidado ambiental, crean una nueva cultura empresarial desde la perspectiva social, convirtiéndose en un nuevo paradigma, tanto para grandes como para pequeñas empresas, pero a éstas últimas se les ha dificultado en gran medida integrarla a la operación del negocio. Es en este sentido que este documento da a conocer los resultados de la investigación que tuvo como objetivo identificar las dificultades que las Pequeñas y Medianas Empresas (PyME) tienen para cumplir con las dimensiones de la RSE: ética, calidad de vida, cuidado ambiental y comunidad; considerando el hecho de que éstas no están aún preparadas, para asumir voluntariamente el paradigma de la responsabilidad social. El enfoque de la investigación fue cualitativo, utilizando el caso de estudio como estrategia de investigación y la entrevista semi-estructurada como método de recolección de datos. El nivel de la investigación fue exploratorio, abriendo con ello el camino para profundizar sobre el tema en futuras investigaciones. Los resultados expresan que la PyME tiene múltiples problemas que deben ser resueltos a partir de mejorar los mecanismos para que la cultura de la RSE sea asumida los miembros y propietarios de la empresa, elevando los intereses sociales sobre los intereses económicos.

Palabras clave: empresa, Responsabilidad Social Empresarial, PyME. 


\title{
THE SME AND THE SOCIALLY RESPONSIBLE COMPANY DISTINCTIVE (ESR): REASONS TO LOSE CEMEFI CERTIFICATION
}

\begin{abstract}
The study on small businesses has long been a relevant issue from various disciplines, due to the importance that this type of organizations has in the social and economic development of the countries. However, it is still necessary to expand their knowledge regarding issues such as competitiveness, innovation, quality and in particular on Corporate Social Responsibility (CSR); whereas the latter assumes broader implications for the impacts of business operations on its environment. The emergence of sustainability and environmental care, create a new business culture from a social perspective, becoming a new paradinegm, both for large and small businesses, but the latter have found it very difficult to integrate it into the operation of the deal. It is in this sense that this document discloses the results of the research that aimed to identify the difficulties that Small and Medium Enterprises (SME) have to meet the dimensions of CSR: ethics, quality of life, environmental care and community; considering the fact that they are not yet prepared, to voluntarily assume the social responsibility paradigm. The research approach was qualitative, using the case study as a research strategy and the semi-structured interview as a method of data collection. The level of research was exploratory, thereby opening the way to deepen the topic in future research. The results express that SME have multiple problems that must be solved by improving the mechanisms for CSR culture to be assumed by the members and owners of the company, raising social interests over economic interests.
\end{abstract}

Keywords: company, Corporate Social Responsibility, SME.

\section{Capítulo resultado de un proyecto de investigación culminado.}

\section{Introducción}

Las operaciones de toda empresa, cualquiera que sea su función, tienen impactos en el medio ambiente interno y externo donde se desenvuelven; y es por ello que deben considerar que sus actividades afectan, positiva o negativamente, la calidad de vida de sus empleados y de las comunidades (Barroso, 2008). La visión de que las empresas deben responsabilizarse de sus actos no es nueva sin embargo, son pocas las que modificaron el paradigma económico a uno más social. Esta nueva perspectiva implica que las empresas asuman una cultura de la Responsabilidad Social (RS), y la incorporen a sus procesos de gestión, para que conformen integralmente las estrategias del negocio y su sistema de planeación (Vives \& Peinado, 2011). Dicho propósito ha sido de gran interés para organismos internacionales como la Organización de las Naciones Unidas (ONU), quien 
desde hace tiempo busca este cambio de paradigma en el mundo, y de manera particular, en las empresas, para lo que la Responsabilidad Social Empresarial (RSE) surge, pero aún los esfuerzos son insuficientes, por lo que pretendiendo impulsar el logro de este objetivo, nacen los sistemas de certificación en responsabilidad social para que las organizaciones sean Empresas Socialmente Responsables (ESR) y generar una cultura socialmente responsable en los negocios (Celis, Torres \& Castro, 2012).

Incursionar en la RSE no es sencillo para ninguna empresa, debe iniciar con un cambio en la cultura de propietarios y empleados, para posteriormente efectuar cambios radicales que van desde la filosofía del negocio hasta las mínimas actividades que se efectúan en todos las áreas funcionales (Barroso, 2008). Es así que de acuerdo con el modelo de gestión del Centro Mexicano para la Filantropía (CEMEFI), las empresas socialmente responsables deben cumplir cuatro ámbitos básicos: ética organizacional, calidad de vida en el trabajo, compromiso con la comunidad y cuidado del medio ambiente. Quienes logran cumplir con los estándares el CEMEFI aseguran una gestión de ESR. Éste organismo otorga un reconocimiento denominado Distintivo ESR, que debe ser ratificado cada año por los negocios, asegurando que están cumpliendo con dichos estándares y que van mejorando en los niveles de responsabilidad social (Cajica , 2008).

La capacidad de la PyME para ser socialmente responsable y obtener la certificación de ESR otorgada por CEMEFI, está limitada por las características que las empresas de menor tamaño presentan en comparación con las grandes empresas (Vives \& Peinado, 2011). Sin embargo, según estadísticas de CEMEFI, más del sesenta por ciento de las empresas que han obtenido la certificación en México son PyME, pero también en esa misma medida, las empresa que pierden la certificación son, en la mayoría de los casos, las de menor tamaño (CEMEFI, 2016), surgiendo así la pregunta: ¿Cuáles son las dificultades que las PyME tienen para cumplir con los estándares de las dimensiones de RSE: ética, calidad de vida, medio ambiente y comunidad? Es en este sentido que la investigación realizada tuvo como objetivo identificar los problemas que tiene la Pequeña y Mediana Empresa (PyME) para lograr interiorizar las dimensiones de RSE en su gestión, las cuales se evidencian con la finalidad de que sean consideradas para establecer nuevos criterios en los modelos de certificaciones, y en los sistemas regulatorios, para que ayuden a que este tipo de negocios asuman la responsabilidad que tienen con la sociedad que atienden.

El modelo de certificación de CEMEFI ha permitido que las empresas en México y otros países de Latinoamérica logren ostentar dicho distintivo, siendo éste un gran aliento para que la sociedad sepa que algunos negocios están preocupados por cumplir con su RS (Celis, Torres \& Castro, 2012), sin embargo, la problemática planteada, fundamentada en las estadísticas de CEMEFI, asegura que la realidad es otra, muchas de las empresas que obtienen el distintivo, lo pierden al paso del tiempo, y el conocimiento que se tiene de por qué las PyMe dejan de ratificar dicha certificación es limitado.

En el caso de México, las causas que motivan a las empresas a dejar de obtener la certificación de ESR otorgado por el CEMEFI, se debe al incremento de los indicadores utilizados para evaluar a las empresas, y a que dejan de recibir los apoyos de las grandes empresas u organismos que los subsidian, sin embargo, no se ha realizado un estudio que profundice en los motivos estructurales que tomen en cuenta las dimensiones de la RSE 
para establecer criterios que ayuden a mejorar los modelos de gestión de RSE, adecuándolas a las características de la PyME y permitiéndoles resolver los problemas que tienen para asumir su responsabilidad social (Reyes, 2017).

\section{Fundamentación teórica}

Los mercados globales han planteado nuevos retos para las empresas, destacando esencialmente el de su persistencia frente a la competitividad. Esta competitividad ha implicado establecerse más allá de su estricta lógica de generación de riqueza, así como de unión con la innovación y el empleo (Andrade, Ramírez, Mendoza \& Sánchez, 2018), a la reorientación de su rol como agentes de cambio y de desarrollo de las comunidades en las cuales se desenvuelven (Vives, Corral \& Isúsi, 2005), entendiendo que es importante se incluya en la gestión el tema de la sustentabilidad social y ambiental. A la sustentabilidad se le ha relacionado con el uso eficiente de los recursos internos para generar ventajas competitivas, así como con el cuidado del uso de recursos externos. Para lograr la sustentabilidad, se requieren estrategias que equilibren lo económico, social y ambiental; y se implementen en la gestión de los negocios (Velázquez \& Vargas , 2012).

La RSE ofrece una nueva opción de gestión estratégica, así como también dirige el compromiso que asumen las empresas hacia la sociedad en beneficio del desarrollo sostenible, es decir, pretende equilibrar el crecimiento económico y el bienestar social sostenible en un contexto complejo y sensible (Sarmiento, 2011). El término de responsabilidad social fue creado para unir a las empresas con el tema de la sociedad y el cuidado del medio ambiente, pretendiendo que los negocios tengan propósitos que vayan más allá de las cuestiones estrictamente relacionadas con la maximización de las utilidades. De esta manera, el concepto de RS se encuentra especialmente relacionado con la legitimidad de la conducta empresarial, desde la perspectiva de su obligación legal hasta el cumplimiento de los intereses sociales que le plantean sus partes interesadas o stakeholders (Aguilera, 2012). Ello implicaría abrir un camino hacia la administración del desarrollo social en concordancia con la competitividad, integrándose a toda la organización en la práctica de sus relaciones (FOMIN, 2009). Los cambios en las organizaciones implica modificar la cultura y la visión, y de ser el principal propósito la obtención de utilidades, se suma el bienestar de sus públicos de interés internos y externos (Cajica , 2008). La RSE se ha exigido en primera instancia a las grandes empresas, a quienes se les ha conminado primordialmente a responder rápidamente a las exigencias actuales de la sociedad, mientas que a las pequeñas se les ha dejado ser, debido a que se piensa que este tipo de negocios no alcanzan los estándares de riesgo ambiental, sin embargo, esta forma de ver a la pequeña empresa es un error desde la perspectiva de la sustentabilidad.

Según datos estadísticos, en el mundo más del 90\% de las empresas son negocios pequeños, participando con un gran porcentaje en el Producto Interno Bruto (PIB) de las naciones, siendo de esta forma, consideradas en muchas regiones, el principal motor de la economía. En el caso de México, según datos estadísticos existen aproximadamente 5.6 millones de unidades económicas instaladas, de éstas el $99.8 \%$ son consideradas micro, 
pequeñas y medianas empresas; aportando el 52\% al PIB y generando el 70\% del empleo en el país (INEGI, 2018). Estos datos explican que de manera particular una pequeña empresa podría no tener mayores impactos al medio ambiente y a la sociedad, pero en conjunto, todas ellas logran tener un impacto muy importante en la sociedad y el medio ambiente. Por todo ello, no puede ser menospreciado su impacto, ni deben ser relevadas de su responsabilidad social, así como de las exigencias sobre el cumplimiento de protocolos que las haga ser más responsables, ya que éstas forman parte del desarrollo y crecimiento sustentable de una nación (Pérez, 2008).

Cox (2011) en su investigación realizada en España, demostró que hay una gran diferencia de comprensión sobre RSE entre las grandes y pequeñas empresas. Para los grandes negocios puede ser más sencillo identificar sus impactos e implementar sistemas y procesos que mejoren sus estándares de responsabilidad social; pero para las empresas de menor tamaño es más complicado, primordialmente porque para los directivos es menos atractivo implementar sistemas de responsabilidad ya que no verá sus beneficios a corto plazo. Y aunque se piensa que la PyME desconoce estas nuevas exigencias de la sociedad, se sabe que éstas no están ciegas a los impactos que causan sus actividades. Es por ello, que se considera un reto para el estudio de las organizaciones encontrar mecanismos de gestión que la PyME pueda implementar equilibrando sus intereses económicos con los de tipo social y ambiental (Vives \& Peinado, 2011).

En este ámbito, países como México desde hace ya varios años han asumido la responsabilidad de atender asuntos de cuidado ambiental exigidos por organizaciones como la ONU en las cumbres mundiales. Poco a poco se ha visto que las organizaciones de este país se han preocupado por cumplir con exigencias sobre la RSE, contando para ello con el Centro Mexicano para la Filantropía (CEMEFI), organismo no lucrativo y no gubernamental que pretende motivar la inclusión de la empresa a la dinámica de la RSE. Esta organización definió algunos principios para que las empresas obtuvieran una certificación, para reconocerles el esfuerzo que realizan sobre acciones socialmente responsables (Porton \& Castromán, 2006).

Es así que dicho organismo une esfuerzos con otras organizaciones que buscan el mismo propósito, con la finalidad de incursionar en las entidades del país; como es el caso del estado de Sinaloa que, a través de AccionaRSE, las empresas pueden conocer los principios del Pacto Global y asumir las responsabilidades que tienen con la sociedad. Así mismo, este propósito es apoyado por el Consejo para el Desarrollo Económico de Sinaloa (CODESIN), que funge como enlace ante AccionaRSE para promover, capacitar y apoyar a las empresas sinaloenses, sin importar su tamaño, y que tienen el interés de cumplir con lo necesario para ser certificadas por el CEMEFI y obtener el Distintivo de ESR (Empresa Socialmente Responsable) pretendiendo con ello que se les reconozca el esfuerzo que realizan sobre este tema (CEMEFI, 2016).

Una certificación implica que las empresas asumen la responsabilidad de cumplir con los estándares mínimos solicitados para que sean reconocidas en lo que se está certificando. En el caso de las certificaciones en RSE, implica mínimamente cumplir con estándares relativos a las dimensiones que se establecen básicas para el cumplimiento de la responsabilidad social en las empresas. 


\section{Dimensiones de la RSE}

- Ética. Se refiere al desempeño ético de la empresa ante sus grupos de interés. Los instrumentos básicos de la ética son los valores, derechos, obligaciones, reglas y relaciones (Stoner, Freeman \& Gilbert, 1996). En algunos casos se requiere revisar los fundamentos legales, para identificar acciones ilegales y que van en contra de la sociedad (Kloter \& Keller, 2012). Es así que la ética no es ajena a la empresa, y por lo tanto, ésta requiere definir sus valores y códigos de conducta a las que se unirán sus empleados (Robbins \& Coulter, 2005).

- Calidad de vida. Implica el compromiso y acciones de la empresa dirigidas a potenciar el desarrollo de sus colaboradores, en un entorno laboral que propicie su empoderamiento y la calidad de vida. Las prácticas laborales se refieren a las relaciones que la empresa debe establecer con sus trabajadores, considerando acciones justas de remuneración, evitar las discriminaciones, entre otros aspectos (Ebert \& Griffin, 2005).

- Cuidado y preservación del medio ambiente. Se enfoca a las actividades relativas a utilizar de forma sustentable y responsable los recursos, al tiempo que previene riesgos y reduce los impactos ambientales. La empresa debe medir la consecuencia de sus operaciones, uniéndose al desafío mundial, de lograr que se desarrolle una economía global sustentable que sea capaz de soportar el planeta (Hart, 1997).

- Comunidad. Constituye las acciones que demuestran el compromiso de la empresa con el desarrollo de la sociedad y el entorno con el que se relaciona. El conocimiento de la comunidad y sus características es fundamental para desarrollar con éxito cualquier empresa para contribuir con la localidad, realizando prácticas ambientales, políticas sobre ética, asuntos relativos al trabajo y la familia, temas de salud, entre otros (Villalobos, 2004).

- Estudios sobre la PyME aseguran, que las características de este tipo de negocios ayudan a que sea más sencillo la ejecución de las dimensiones de RSE, precisamente por la flexibilidad en sus operaciones, a que sus canales de comunicación son más rápidos y sus estructuras organizacionales menos complejas (Margolis \& Walsh, 2001), pero sus debilidades como la limitada disposición de recursos, la dificultad para encadenarse a sistemas productivos con otras empresas más grandes, sus deficiencias para realizar planes, diseñar estrategias, entre otras, evita que la PyME pueda ejecutar acciones de responsabilidad social a largo plazo (Jenkins, 2004).

\section{Metodología}

El estudio se realizó bajo el enfoque cualitativo, utilizando para la recolección de datos una entrevista semi-estructurada, misma que fue diseñada en base a la encuesta de Ethos y aplicada a empresas de tamaño pequeño y mediano que obtuvieron la certificación de ESR otorgado por CEMEFI en México, pero la perdieron en menos de tres años, bajo la pretensión de que para conocer los problemas del cumplimiento de las dimensiones, las empresas debieron haber experimentado su desarrollo. Con base en lo anterior, se justifica 
el caso de estudio como la estrategia de investigación, tomando en cuenta que era necesario sortear la dificultad de rechazo de las empresas, por lo que se seleccionaron a juicio del investigador, cumpliendo con los criterios que se muestran en la siguiente tabla 1.

Tabla 1: Criterios para la selección de los Casos de Estudio.

\begin{tabular}{cc}
\hline Criterio & Justificación \\
\hline Haber estado afiliada a CEMEFI & $\begin{array}{r}\text { Es evidencia que aplicaron en su empresa un modelo } \\
\text { de gestión de RSE. }\end{array}$ \\
\hline
\end{tabular}

Haber perdido la certificación de ESR

Es el objeto de análisis.

\begin{tabular}{|c|c|}
\hline Ser de tamaño PyME & $\begin{array}{l}\text { Son la mayoría de empresas que han perdido el } \\
\text { Distintivo de ESR. }\end{array}$ \\
\hline $\begin{array}{c}\text { Motivos para obtener el Distintivo de } \\
\text { ESR }\end{array}$ & $\begin{array}{l}\text { Contó con un propósito para ser una empresa } \\
\text { socialmente responsable. }\end{array}$ \\
\hline Años de actividad & $\begin{array}{l}\text { Es una empresa instalada con por lo menos } 5 \text { años en } \\
\text { el mercado. }\end{array}$ \\
\hline Número de empleados & $\begin{array}{c}\text { Por lo menos cuenta con } 10 \text { empleados y } 50 \text { como } \\
\text { máximo. }\end{array}$ \\
\hline
\end{tabular}

Fuente: elaboración propia (2019).

\section{Casos seleccionados}

La selección de los casos estudiados cuidó el cumplimiento de las limitaciones y criterios señalados, eligiéndose para llevar a cabo el trabajo de campo a cuatro empresas, en base a que se afirma que para estudios cualitativos es adecuado trabajar en un rango de cuatro a diez casos de estudio, ya que menos de cuatro casos empíricamente no es conveniente por (Martines, 2006), sin embargo, otros autores como (Perry, 1998), (Patton, 1990), señalan que la decisión de la cantidad está en el criterio del investigador. Los Casos de estudio además de cumplir con los criterios y delimitaciones establecidos, se eligieron por conveniencia en la diversidad de giros, años en el mercado y motivos que tuvieron para decidir obtener el Distintivo de ESR otorgada por CEMEFI. 
Para lograr el objetivo de la investigación, fue necesario que el nivel de la investigación fuera exploratorio, ya que este tema no ha sido estudiado a profundidad en todos sus factores. Además, ayudó a elegir adecuadamente a los casos de estudio según la estrategia de investigación elegida para obtener los datos que permitirían resolver la problemática planteada. Como estudio exploratorio, se eligió al estado Sinaloa para ser la entidad a estudiar, y según el criterio de disponibilidad, se seleccionó a la ciudad de Los Mochis, tomando en cuenta que ésta es la segunda ciudad que registra una cantidad importante de negocios PyME que han perdido el distintivo de ESR otorgada por CEMEFI, como se muestra en la siguiente tabla 2.

Tabla 2: Porcentaje de empresas que han perdido el distintivo ESR en Sinaloa según su tamaño durante el periodo del 2014 al 2108.

\begin{tabular}{cccccc}
\hline Ciudades & Micro & Pequeña & Mediana & Grande & TOTAL \\
\hline Los Mochis & $6.8 \%$ & $2.3 \%$ & $13.6 \%$ & $2.3 \%$ & $25 \%$ \\
\hline Guasave & 0 & 2.3 & 0 & 0 & $2 \%$ \\
\hline Culiacán & $4.6 \%$ & 18 & $20.5 \%$ & 0 & $43 \%$ \\
\hline Mazatlán & 0 & 4.6 & $13.6 \%$ & 0 & $18 \%$ \\
\hline Otras ciudades & 0 & 4.6 & $6.8 \%$ & 0 & $12 \%$ \\
\hline Total & 11.4 & 31.8 & 54.5 & 2.3 & $100 \%$ \\
\hline
\end{tabular}

Fuente: CEMEFI, (2018); ACCIONARSE, (2018).

En total hay 329 empresas certificadas en Sinaloa hasta el año 2018; el 25\% están instaladas en la ciudad de Los Mochis, el 2\% en Guasave, el 43\% se localizan en Culiacán, un $18 \%$ están en Mazatlán y el $12 \%$ se encuentran distribuidas por el resto de las ciudades. En las ciudades de Los Mochis, Culiacán y Mazatlán se concentra la mayoría de las empresas que han dejado de ratificar la certificación como ESR, y como se puede observar en la tabla anterior la mayoría son de tamaño mediano (54\%) y pequeñas $(31.8 \%)$, en menor número las micro-empresas (11.4\%), dejando en último lugar a las grandes empresas $(2.3 \%)$ que se han mantenido con el distintivo por más tiempo.

La recolección de datos consistió en la aplicación de entrevistas a todos los empleados, directivos y propietarios de cada empresa (ver tabla 3), guiándose la investigación por el supuesto de que la poca claridad en los planes de RSE, las limitaciones financieras, el desinterés de los propietarios y empleados y la desarticulación de la estrategia con las dimensiones de RSE: ética, calidad de vida, medio ambiente y comunidad, son problemas que limitan a la PyME asumir una cultura socialmente responsable a largo plazo. 
Tabla 3: Público objetivo en los casos de estudio.

\begin{tabular}{cc}
\hline Público & Información solicitada \\
\hline Propietarios / Gerentes & $\begin{array}{c}\text { Conocimiento sobre la empresa, la filosofía, motivos de la } \\
\text { certificación y pérdida del Distintivo de RSE, proceso de gestión de } \\
\text { la RSE. }\end{array}$ \\
\hline Jefes de área & Información sobre la aplicación de las dimensiones de ESR. \\
\hline Empleados & $\begin{array}{c}\text { Opinión sobre la ejecución real de las dimensiones de ESR en la } \\
\text { empresa y su inmersión en la cultura de RSE. }\end{array}$ \\
\hline
\end{tabular}

Fuente: elaboración propia (2019).

El público objetivo ofreció información relacionada con los propósitos de la investigación, misma en la que se basó para la elaboración de los reactivos, análisis e interpretación de los resultados. De igual manera fue posible que a partir de los resultados de la investigación, producto de la interpretación de los datos obtenidos, éstos fueron validados con la observación in situ y la revisión documental de hechos registrados en las empresas estudiadas.

\section{Resultados}

Los resultados cumplieron con el objetivo de la investigación, al lograr identificar los problemas que la PyME tiene para cumplir con las dimensiones de la RSE: ética, calidad de vida, cuidado ambiental y comunidad, mismas que se muestran en la tabla 4.

Tabla 4: Problemas de la PyME para mantener la certificación de ESR del CEMEFI.

\begin{tabular}{|c|c|c|c|}
\hline Sobre la ética & $\begin{array}{c}\text { Sobre calidad } \\
\text { de vida }\end{array}$ & Sobre la comunidad & $\begin{array}{c}\text { Sobre el medio } \\
\text { ambiente }\end{array}$ \\
\hline $\begin{array}{l}\text { No se dan a conocer } \\
\text { a los miembros } \\
\text { de la empresa, y } \\
\text { a su público de } \\
\text { interés los valores } \\
\text { y principios éticos } \\
\text { que guiarán sus } \\
\text { decisiones. }\end{array}$ & $\begin{array}{c}\text { No incluyen a los } \\
\text { colaboradores en } \\
\text { el diseño de los } \\
\text { planes, programas y } \\
\text { estrategias. }\end{array}$ & $\begin{array}{l}\text { No desarrolla políticas, } \\
\text { planes y programas que } \\
\text { demuestres interés por } \\
\text { su público externo, más } \\
\text { allá del beneficio que de } \\
\text { ellos puede obtener. }\end{array}$ & $\begin{array}{l}\text { No existe integración } \\
\text { de colaboradores } \\
\text { y propietarios } \\
\text { para reafirmar } \\
\text { compromisos de RSE } \\
\text { medioambientales, } \\
\text { resultando en acciones } \\
\text { débiles sobre este } \\
\text { ámbito. Las decisiones } \\
\text { están basadas en los } \\
\text { costos y beneficios para } \\
\text { competir en precios. }\end{array}$ \\
\hline
\end{tabular}


Tabla 4: Problemas de la PyME para mantener la certificación de ESR del CEMEFI (Continuación).

\begin{tabular}{|c|c|c|c|}
\hline Sobre la ética & $\begin{array}{c}\text { Sobre calidad } \\
\text { de vida }\end{array}$ & Sobre la comunidad & $\begin{array}{c}\text { Sobre el medio } \\
\text { ambiente }\end{array}$ \\
\hline $\begin{array}{l}\text { No establecen } \\
\text { lineamientos o } \\
\text { políticas que den } \\
\text { seguimiento a los } \\
\text { asuntos éticos. }\end{array}$ & $\begin{array}{l}\text { No cuentan con } \\
\text { herramientas que } \\
\text { fortalezcan la } \\
\text { capacitación y } \\
\text { formación de los } \\
\text { colaboradores, } \\
\text { considerándolo } \\
\text { un gasto para la } \\
\text { empresa. }\end{array}$ & $\begin{array}{c}\text { No cuenta con } \\
\text { sistemas formales } \\
\text { de comunicación } \\
\text { para conocer a sus } \\
\text { clientes, proveedores, } \\
\text { intermediarios y } \\
\text { habitantes en general, } \\
\text { de donde pueda recibir } \\
\text { sugerencias o críticas. }\end{array}$ & $\begin{array}{l}\text { No genera políticas, } \\
\text { ni programas sobre } \\
\text { el uso de materiales } \\
\text { contaminantes } \\
\text { (empaques, envases, } \\
\text { etc.), producto de sus } \\
\text { operaciones, uso de } \\
\text { desperdicios, proceso } \\
\text { de reciclaje, entre } \\
\text { otros. Desconoce cómo } \\
\text { medir los impactos } \\
\text { ambientales de sus } \\
\text { actividades. }\end{array}$ \\
\hline $\begin{array}{c}\text { No existen } \\
\text { programas en donde } \\
\text { se interiorice a los } \\
\text { miembros de la } \\
\text { empresa los valores } \\
\text { y principios éticos } \\
\text { establecidos. }\end{array}$ & $\begin{array}{c}\text { No existe } \\
\text { preocupación por } \\
\text { diseñar planes } \\
\text { y programas a } \\
\text { largo plazo, donde } \\
\text { se forme a los } \\
\text { colaboradores en } \\
\text { asuntos de la RSE } \\
\text { para consolidar este } \\
\text { tema en la empresa. }\end{array}$ & $\begin{array}{l}\text { No da a conocer a su } \\
\text { público externo las } \\
\text { acciones socialmente } \\
\text { responsables que realiza } \\
\text { o tiene programadas } \\
\text { realizar, y si lo hace es } \\
\text { de forma limitada. }\end{array}$ & $\begin{array}{l}\text { No muestra conciencia } \\
\text { sobre el daño de sus } \\
\text { actividades al medio } \\
\text { ambiente. Son más } \\
\text { importante las ganancias } \\
\text { o los bajos costos. }\end{array}$ \\
\hline $\begin{array}{l}\text { No se observa que } \\
\text { los propietarios y } \\
\text { directivos les den } \\
\text { real importancia a } \\
\text { los asuntos éticos. }\end{array}$ & & $\begin{array}{l}\text { No establece programas } \\
\text { de colaboración } \\
\text { conjunta con miembros } \\
\text { de la comunidad. }\end{array}$ & $\begin{array}{l}\text { Los actos de cuidado } \\
\text { ambiental se limitan } \\
\text { a acciones sociales de } \\
\text { limpieza, conservación } \\
\text { de áreas, reutilización de } \\
\text { papel o empaques, venta } \\
\text { de materiales, entre } \\
\text { otros, sin ir más allá con } \\
\text { visión de largo plazo. }\end{array}$ \\
\hline $\begin{array}{c}\text { No miden el } \\
\text { impacto que tiene } \\
\text { en el público } \\
\text { de interés, las } \\
\text { acciones éticas de la } \\
\text { empresa. }\end{array}$ & & $\begin{array}{l}\text { No muestra iniciativa } \\
\text { por impulsar } \\
\text { ante organismos, } \\
\text { instituciones, gobierno, } \\
\text { etc., acciones que } \\
\text { reditúen en beneficio de } \\
\text { la propia comunidad, } \\
\text { que genere entre } \\
\text { todos una cultura de } \\
\text { responsabilidad social. }\end{array}$ & $\begin{array}{l}\text { No atiende a las } \\
\text { exigencias legales sobre } \\
\text { el impacto ambiental, } \\
\text { debido a que éstas en } \\
\text { lo individual, están } \\
\text { fuera de los límites } \\
\text { establecidos por la ley. }\end{array}$ \\
\hline
\end{tabular}

Fuente: elaboración propia (2019). 
Así mismo, se lograron identificar las dificultades que limitan su gestión al implementar los planes, estrategias y actividades relativas a la RSE, y que se dan a conocer a continuación:

Los anteriores problemas se complementan con otro tipo de limitaciones relativas a la forma de cómo operan las PyME, y son importantes darlas para mayor claridad sobre las dificultades que sufren este tipo de negocios para desarrollarse como empresa socialmente responsable.

\section{Dificultades de las PyME para efectuar una adecuada gestión de RSE}

Algunas de las principales dificultades identificadas en las PyME para que su gestión se alinee con las dimensiones de la RSE son las siguientes:

\section{Dificultad en la estructura organizacional de la empresa:}

La PyME no se preocupa por diseñar puestos o áreas específicas que se hagan responsables de la RSE dentro de la empresa. La ejecución de estas actividades se suma a las de los puestos que son afines, y a quienes se les responsabiliza le da más prioridad a las funciones de su puesto. El personal no se encuentra comprometido, debido a que su participación es voluntaria.

\section{Dificultad financiera:}

La PyME no cuenta con recursos financieros adicionales o exclusivos para llevar a cabo y conservar actividades de RSE. Este tipo de negocios tiene recursos económicos limitados, por lo que no asigna un presupuesto a las actividades de RSE, y cuando lo hace es limitado, por corto tiempo, o no es constante. Buscan apoyos externos, y cuando los obtienen, se atienen a ellos, y cuando los pierden, también pierden el interés por continuar con las actividades de RSE. Es el caso de los apoyos que reciben de las grandes empresas al participar en la cadena de valor, o los que reciben de organismos públicos. Los propietarios consideran que todas las acciones relativas a la RSE son un costo a corto plazo, no lo ven como una inversión a largo plazo con beneficios no sólo económicos, sino también sociales.

\section{Poca claridad en planes y estrategias de RSE:}

La PyME que ha obtenido el Distintivo de RSE, si bien cuenta con una misión, visión, objetivos, código de ética, entre otros documentos formales, los planes relativos a la RSE se diseñan con poca visión de sustentabilidad y son a corto plazo. Evitan los estándares de medición de resultados de los programas implementados y/o de sus impactos sociales y ambientales por considerarlos innecesarios. Descuidan los programas que evitan acciones de discriminación, corrupción, impactos ambientales, impactos a poblaciones vulnerables. La inversión social no está contemplada en los planes a largo plazo. 
Los directivos y dueños de la PyME tienen la idea de que no hay beneficios directos entre las acciones de RSE con la reputación, reconocimiento de la comunidad, mejora de imagen ante el mercado, competitividad, entre otros. Los propietarios carecen de conciencia social y cuando si existe en ellos, desconocen cómo transmitirla a sus colaboradores.

Es palpable la poca participación que tienen los colaboradores en el diseño de los planes, programas, metas y estrategias en general y especialmente en lo relativo a la RSE. No logran comprenderlos, lo que limita la interiorización de la cultura de RSE en toda la empresa, y el compromiso con los propósitos sociales de la empresa. Sumándose a ello la poca promoción para que la población participe con ellos en propósitos más amplios que compete a la propia comunidad.

\section{Discusión}

Las certificaciones han sido desde siempre una forma de asegurar el cumplimiento de estándares para lograr un objetivo específico, primordialmente esperado por el mercado o por algún grupo de interés, y para muchos es una forma de lograr que las empresas lleguen a ser competitivas (Guajardo, 2008), sin embargo, también las certificaciones han sido ampliamente criticadas, en virtud de que su propósito se ha desvirtuado, y en el caso de las certificaciones en RSE también son desacreditadas, pero a su vez, han sido un mecanismo útil para que las empresas modifiquen el paradigma hacia la responsabilidad social con el propósito de lograr una mayor sustentabilidad de las empresas en el tiempo, en virtud de que a la RSE se le ha considerado una ventaja competitiva, puesto que le agrega valor a la empresa, sin embargo, la realidad es que los dueños de los negocios no lo ven así.

Y si bien los modelos de RSE pretenden aportar mecanismos que faciliten a los negocios atender las exigencias y expectativas de convertirse en empresas socialmente responsables, éstos implican que la PyME en particular, deba realizar más esfuerzos que las grandes empresas para atender las exigencias, requiriendo usar más recursos que los que normalmente utiliza y realizar mayores cambios, mismos que no está dispuesta a asumir, regresando a la situación de siempre, y manteniendo el paradigma económico como su único objetivo.

Para que la RS sea operativa y aplicable a los procesos de gestión de las organizaciones se requiere alentar a que tanto las organizaciones como los gobiernos asuman su responsabilidad en sus desempeños internos y externos, para que la empresa logre ese crecimiento sostenible e integrador que establece las bases para atender las cuestiones de la RSE (COM, 2010). No obstante los benéficos que representa para las empresas atender los aspectos relacionados con responsabilidad social, existe aún incertidumbre en que este nuevo paradigma puede ser parte de la gestión de pequeñas empresas. Por lo tanto existe la necesidad de que se resuelvan esos problemas para alcanzar el objetivo de una verdadera sostenibilidad del modelo de desarrollo socioeconómico. 
Aunado a lo anterior, es necesario reconocer la existencia de posturas en donde se señala que la RS en las organizaciones no es sostenible porque tiene un costo, y que las certificaciones en RSE es una simulación que no ayuda a que se logre la interiorización de ésta en la empresa para cumplir con los principios de ser socialmente responsable (Rochiln, 2005). Sin embargo, los beneficios deben ser palpables, los esfuerzos deben dar frutos, y los resultados se verán a largo plazo, por lo que mantener una postura positiva hacia las acciones dirigidas a la RS es necesaria, sin importar la forma de cómo se lograría, si con las certificaciones que motivan la acción voluntaria, o con las leyes que exigen acciones obligatorias.

Cualquiera que sea la dirección a tomar, para lograr que las empresas dirijan sus pasos hacia la RSE es recomendable que se tomen en cuenta las características de cada tipo de negocio, se consideren sus propios estándares, estructura, visión del propietario y el ámbito local en el que operan (Guibert, 2009), siendo imprescindible la actuación desde una perspectiva sistémica, orientada precisamente hacia el cambio, teniendo en cuenta que los negocios son parte importante del problema, y por ende, también deben serlo de la solución, los niveles de acción deben ser más visionarios y proactivos para lograr un verdadero cambio a largo plazo en el tema de la sustentabilidad social y ambiental.

\section{Consideraciones Finales}

Las dificultades que la PyME tiene para cumplir con las dimensiones de la RSE: ética, calidad de vida, cuidado ambiental y comunidad, surgen por problemas a partir de la identificación de factores clave que influyen en las dimensiones de la RSE. Sin embargo, para enfrentar el problema que presenta la PyME ante la falta del cumplimento a las dimensiones de RSE es necesario un cambio en la forma de pensar del mundo entero, un cambio de gestión en torno a la sustentabilidad, al cuidado del medio ambiente y a aspectos relativos a la ética, a la calidad de vida de las personas dentro y fuera de los lugares de trabajo, y al desarrollo social de las comunidades. Así como cambiar las conductas de manera profunda en la mentalidad de los directivos de los negocios o de los propietarios, y en este sentido es que algunas empresas, que ya han obtenido las certificaciones y han iniciado el cambio hacia la sustentabilidad, en un momento determinado, ya sea uno o diez años, deja de ser un asunto importante, y pierden las certificaciones.

A diferencia de las grandes empresas, las pequeñas desisten de sus intenciones de seguir el camino de la RSE después de los tres años, lo cual es preocupante, ya que las empresas de menor tamaño tienen mayor contacto con la comunidad, la relación con sus grupos de interés es más estrecho, y por el número de éstas en el mundo, el impacto es mayor en el desarrollo social de las regiones y localidades. Se requiere que el CEMEFI establezca estrategias diferentes que ayuden, y no limiten a la PyME a mantener las certificaciones en RSE, disminuyan sus debilidades, desconocimiento, falta de interés, y se establezcan acciones que las obliguen a reconocer y a actuar en pro de formar parte de las organizaciones responsables de los impactos de sus actividades. Que valoren la importancia que tiene lograr el Distintivo de ESR por los beneficios que esto puede tener en la disminución de sus costos, ampliar sus mercados, incluir sus operaciones en cadenas productivas, optimizar su imagen ante la comunidad y mejorar el medio ambiente en donde se desarrolla. Así como 
también es necesaria la colaboración del estado, instituciones educativas y organizaciones, imperando para este propósito continuar con investigaciones que profundicen en la identificación de problemáticas y de sus soluciones.

\section{Referencias bibliográficas}

Aguilera, A. (2012). Pensamiento y gestión. (32), 26. Recuperado el 20 de Enero de 2017, de http://www.scielo.org.co/pdf/pege/n32/n32a02.pdf

Andrade, J., Ramírez, E., Mendoza, J., \& Sánchez, H. (2018). La innovación, el capital intelectual y la competitividad en las pequeñas empresas de sur colombiano. En Y. Chirinos, A. Ramírez, N. Barbera, \& L. Camejo, Tendencias en la investigación universitaria. Una visión desde Latinoamérica. (págs. 51-66). Venezuela: Fondo Editorial Universitario Servando Garcés.

Barroso, F. (2008). La responsabilidad social empresarial un estudio de cuarenta empresas de la ciudad de Merída Yucatán. Contaduria y Adminstración(226), 20.

Cajica , J. (Julio de 2008). El concepto de responsabilidad social empresarial. Obtenido de CEMEFI: https://www.cemefi.org/esr/images/stories/pdf/esr/concepto_esr.pdf

Celis, L., Torres, A., \& Castro, M. (2012). Analizando las normas en que se basan las acciones de responsabilidad social empresarial (RSE) en México. Contabilidad y Auditoria, 28.

CEMEFI.(2016).CentroMexicanoparala Filantropia.Obtenido dewww.cemefi.org:https:// www.cemefi.org/esr/images/.../convocatorias2017/2017 convocatoriaPyME. pdf

COM. (2010). Una estrategia para un crecimiento inteligente, sostenible e integrador. Comision Europea. Obtenido de www.aragon.es/estaticos/GobiernoAragon/ Departamentos/HaciendaAdministracionPublica/Documentos/docs2/Areas/ Fondos\%20Estruct\%20UE/2014-2020/COM(2010)2020-Europa2020Unaestrategiaparauncrecimiento.pdf

Cox, R. (2011). Responsabilidad Social Empresarial de las Pymes: El caso del distrito V de Huelva España. (C. d. Investigación, Ed.) Organizacion y Sociedad, 18(58). Recuperado el 5 de Abril de 2016

Ebert, R., \& Griffin, R. (2005). Business Esssentials. (7a ed. ed.). U.S.A: Pearson Printice Hall.

FOMIN. (2009). Guía de aprendizaje sobre la implementación de Responsabilidad SociL Empresarial en pequeña y medianas empresas . MIF FOMIN.

Guajardo, E. (2008). Administración de la calidad total. México: Pax México.

Guibert, U. (2009). Responsabilidad Social Empresarial: competitividad y casos de buenas práticas en Pyme. Google Scholar. 
Hart, S. (1997). Beyond greening.estrategies for a sustainable word. Havard DBussiness Review, 75.

INEGI. (30 de julio de 2018). Censos Economicos 2014. Obtenido de www.inegi.org.mx

Jenkins, H. (2004). A Critique of convertional CSR Theory: An SME Perspective. Journal of general Management, 29(4).

Kloter, P., \& Keller, K. (2012). Direccón de Marketing. (D. C. Guillermo, Ed.) México: Pearson Educación.

Margolis, D., \& Walsh, J. (2001). People and Profits? the search for a link between a company's social and financial performance / Joshua Daniel Margolis, James Patrick Walsh (3era ed.).

Martines, M. (2006). La investigación Cualitativa (sintesis Conceptual). IIPSI, 9(1). Obtenido de http://sisbib.unmsm.edu.pe/bvrevistas/investigacion_psicologia/ v09_n1/pdf/a09v9n1.pdf

Patton, M. (1990). Qualitative evaluation and research methods. SAGE Publications, inc.

Perez, M. (2008). Piensa Colombia. Hacia el desarrollo Sostenible en Colombia (Vol. 3). (U. N. Manizales, Ed.) Colombia: Colombia. Obtenido de https://www. researchgate.net/publication/317905432_Hacia_el_desarrollo_sostenible_en_ Colombia

Perry, C. (1998). Processes of a case study methodology for postgraduate research in marketing. European journal of marketing, 32.

Porton, N., \& Castromán, J.(2006). Responsabilidad social: un analisis de la situacion actual en Mexico y España. Contaduria y Administración(220), 67-88. Recuperado el 16 de Mayo de 2017, de http://www.redalyc.org/pdf/395/39522004.pdf

Reyes, F. (2017). Las Pymes. (E. Cota, Entrevistador) Culiacan, Sinaloa, México. Recuperado el 8 de Febrero de 2017

Robbins, S., \& Coulter, M. (2005). Administración (8va ed. ed.). México: Pearson.

Rochiln, S. (2005). Llevar la responsabilidad corporativa al ADN de su empresa. Harvard Business Review, 83(8).

Sarmiento, S. (2011). La responsabilidad social empresarial: gestion estrategica para la supervivencia de las empresas. Dialnet, 9(2), 6-15. Recuperado el 10 de Marzo de 2017, de https://dialnet.unirioja.es/descarga/articulo/3965840.

Stoner, J., Freeman, E., \& Gilbert, D. (1996). Administración (Vol. 6a.ed.). (M. S. Pilarr, Trad.) México: Prentice_Hall. Recuperado el 20 de Abril de 2017

Velázquez , L., \& Vargas , J. (2012). La sustentabilidad como modelo de desarrollo responsable y competitivo. Ingeniería de Recusos Naturales y del Ambiente, 11.

Villalobos, J. (2004). Del dicho al hecho. II conferencia Interamericana de Responsabilidad Social Empresarial. 
Vives, A., \& Peinado, E. (2011). RSE La Responsabilidad Social de la Empresa en America Latina. (A. Vives , \& E. Peinado , Edits.) Recuperado el 18 de Septiembre de 2016, de https://publications.iadb.org/bitstream/handle/11319/5383/ La\%20responsabilidad\%20social\%20de $\% 201$ a $\% 20$ empresa $\% 20$ en $\% 20$ Am\%C3\%A9rica\%20Latina\%20.pdf?sequence=1

Vives, A., Corral, A., \& Isúsi, I. (2005). Responsabilidad Social de la Empresa en las Pymes de Latinoamerica (1 ed., Vol. 1). Whashinton, DC: Banco Iberoamericano de Desarrollo. 